\title{
The Role of Physicians in Assessing Older Drivers: Barriers, Opportunities, and Strategies
}

\author{
Hillary R. Bogner, MD, MSCE, Joseph B. Straton, MD, Joseph J. Gallo, MD, MPH, \\ George W. Rebok, PhD, and Penelope M. Keyl, PhD
}

Background: Evaluation of the older driver is a difficult task for primary care physicians. We investigated the physician-perceived barriers to assessing older drivers in primary care practice.

Methods: Twenty family physicians whose patients had completed a clinical questionnaire and neuropsychological tests participated in one of 2 focus groups. Physicians were asked about barriers to assessing older drivers in primary care and the usefulness of neuropsychological tests for assessing driving ability.

Results: A number of themes emerged related to barriers in the assessment of the older driver. Major themes included concerns about being liable for the results of driving related screening and about patients reacting unfavorably to a driving assessment including cognitive tests. Physicians uniformly agreed that a protocol to guide driving assessment would be useful.

Conclusions: Physicians encounter a number of barriers to assessing older drivers but recognize the importance of driving within the context of geriatric functional assessment. (J Am Board Fam Pract 2004; 17:38-43.)

Driving is the epitome of independence. For adolescents, learning to drive is the gateway to adulthood. For older adults, giving up driving is imbued with the meaning that one's skills and autonomy are on the wane. For this reason, to question one's ability to drive a car is to question one's soundness of mind. The physician often becomes the arbiter in a dispute between a worried family and an older driver. Regulations regarding driving vary from state to state. Physicians can give their opinions regarding driving, but licensing is determined by the state. Yet there are few guidelines for the evaluation of older drivers in the medical office. ${ }^{1}$ Standard tests of cognitive performance, such as the

Submitted, revised 19 May 2003.

From the Department of Family Practice and Community Medicine, University of Pennsylvania School of Medicine, Philadelphia (HRB, JBS, JJG), Department of Mental Hygiene, Johns Hopkins University School of Hygiene and Public Health, Baltimore, Maryland (GWR), and Department of Emergency Medicine, Johns Hopkins University School of Medicine, Baltimore, Maryland (PMK). Address correspondence to Hillary R. Bogner, MD, MSCE, Department of Family Practice and Community Medicine, The University of Pennsylvania, 3400 Spruce Street, 2 Gates Building, Philadelphia, PA 19104 (e-mail: bogner@mail. med.upenn.edu).

Data analysis was supported by an American Academy of Family Physicians Advanced Research Training grant (to $\mathrm{HRB}$ ) and by a Health Resources and Services Administration Faculty Development Program in Primary Care Training grant (to JBS). This study was funded by a grant from the American Association of Retired Persons (to PMK).
Mini-Mental State Examination, ${ }^{2}$ and aspects of the standard clinical examination, such as testing reflexes, do not capture the features of perception and attention that are critical for the safe operation of an automobile. ${ }^{3}$

The investigation presented here represents one phase in a line of research whose purpose is to develop a screening protocol for older drivers that is useful to the primary care physician. ${ }^{3-6}$ The purpose of this study was to consider the barriers faced by primary care physicians when assessing older drivers. No studies have yet examined the perspective of primary care physicians regarding the barriers and opportunities related to the assessment of driving. Our goal was to examine the views of primary care physicians so that we could develop a test battery that not only could predict unsafe driving behavior but also would be feasible for use in a primary care setting.

\section{Methods}

Patients who were aged 65 years and older and still driving were identified in the waiting room of 2 primary care practices. After completing a driving questionnaire assessing driving habits, traffic crashes, violations, and near misses, a number of neuropsychological tests were performed (Table 1). The battery described in Table 1 assesses aspects of attention, visual information processing, and verbal 
Driving Questionnaire: An assessment of where, when, and how often and how far patients drive and a history of the patients' crashes, violations, and 'near misses,' and driving self-appraisal of safety in driving over the two years prior to interview.

Motor-Free Visual Perception Test, Visual Closure ${ }^{19}$ : A multiple-choice test of visual perception. Participants are shown a card with a target figure and are asked to choose, from four choices of incomplete drawings, the one drawing that, if finished, would look like the target figure.

Continuous Performance Task ${ }^{20}$ : The patients are asked to use a test device that shows a screen flashing ' $\mathrm{X}^{\prime}$ s at the rate of about one per second. The task is to indicate that a complete ' $\mathrm{X}^{\prime}$ was seen by pressing a button as rapidly as possible. Reaction time and accuracy are assessed.

Visual Reproduction Test ${ }^{21}$ : This is a test of immediate visual memory in which patients view a line drawing for 10 seconds and then must draw the design from memory. Four drawings are presented, one at a time.

Trail-Making Test, Part $A^{22}$ : The patient is required to connect numbered dots on a page, 1 to 25 , in order. The time to complete the task and the number of errors are recorded.

Standardized Road Map of Directional Sense ${ }^{23}$ : Patients trace a path through a simulated street map consisting of 32 possible right or left turns indicating correct directional judgment.

Brief Test of Attention ${ }^{24}$ : A tape recorder presents a series of interspersed digits and letters. In one condition, patients must count and report the number of digits presented. In the other condition, patients must count the number of letters presented.

Mini-Mental State Examination ${ }^{2}$ : An evaluation of cognitive performance including orientation to time and place, memory, and language.

fluency. These areas of cognitive functioning were targeted because they have been found to relate to performance on a driving simulator, crashes, or driving performance for older persons and are thought to be important for safe driving. This paper focuses on the component of the project related to obtaining feedback from physicians on the usefulness of the results of neuropsychological tests and on their opinions of the barriers to assessing driving ability among older adults in the primary care setting. A previous paper discussed the potential utility of brief neuropsychological measures to assess crash risk in older primary care patients. ${ }^{5}$ Informed consent was obtained from the participants, and the study was approved by the Committee on Human Research of the Johns Hopkins University School of Hygiene and Public Health and by the Institutional Review Board of the Franklin Square Hospital Center.

For this part of the project, focus groups with physicians whose patients were examined were conducted at each site. The focus groups occurred at the Family Health Center of the Franklin Square Hospital Center and the East Point Medical Center in Baltimore, Maryland. The focus groups lasted approximately 1.5 hours. All the physicians were family physicians whose patients were selected in the waiting room of 2 primary care practices for the previous study. ${ }^{5}$ Several questions were posed to focus the discussion in meetings with physicians whose patients were examined, centering around (1) screening for driving ability; (2) factors that raise suspicion that driving ability is impaired; and (3) how neuropsychological tests might be incorporated into clinical practice. Specifically, physicians were initially asked the following open-ended questions:

- "Should you bring up the issue of driving with a patient or family when neither the patient nor the family has brought it up? What are the barriers to doing this?"

- "What is it about a patient that would make you think about their driving ability and risk of crashes?"

- "How would you incorporate results of tests of attention, visual perception, etc, into your evaluation of a patient?"

The physicians were reminded of the content of the neuropsychological tests. Physicians were presented with odds ratios representing the strength of the association between test scores and crash involvement derived from patients in the study. ${ }^{5}$ An example of this information is shown in Table 2. The information we provided was only to elicit physicians' ideas about the usefulness of the tests. The information in Table 2 was given to physicians so they could interpret test results from specific patients from their own practice. Detailed analysis of the relationship between neuropsychological tests in older drivers in primary care and involvement in motor vehicle crashes or near misses can be found elsewhere. ${ }^{5-7}$ Specific examples were se- 
Table 2. Association of Test Scores and Crash Involvement in Primary Care Patients, as Shown to Family Physicians in Focus Groups

\begin{tabular}{lcc}
\hline Test domain & $\begin{array}{c}\text { Odds } \\
\text { Ratio* }^{*}\end{array}$ & $\begin{array}{c}\text { 95\% Confidence } \\
\text { Interval }\end{array}$ \\
\hline Attention & 2.9 & $1.2-6.8$ \\
Visual closure & 1.8 & $0.8-4.0$ \\
Reaction time & 2.1 & $0.3-12.8$ \\
Directional sense & 1.3 & $0.6-3.2$ \\
Trailmaking & 2.1 & $0.9-5.2$ \\
Mini-Mental State & 1.7 & $0.5-5.8$ \\
$\quad$ Examination score $<24$ & & \\
\hline
\end{tabular}

* Odds ratio represents the proportion of patients reporting involvement in crashes or near misses among persons in the poorest quintile of performance for each test compared to patients in the better performing quintiles.

lected from the patients examined in the study and presented, without identifiers, to the physicians for discussion. The format for display of the information from the neuropsychological assessments is shown in the example in Table 3. Results were expressed as a quintile ranking of the patient compared with other patients that were tested at that site. We believed that this would be more readily

Table 3. Format for Display of Results from Patient Testing

A 78-year-old woman with diabetes and hypertension who describes her overall health as 'fair.'

She has been driving for 62 years.

-She reports that she drives anywhere but only during the daytime, in good weather, and not during rush hour. She reports driving between 11 and 50 miles per week for each of four roadway categories: in her neighborhood, in the city, on secondary roads, and on the freeway, for an estimated 6240 miles per year.

-She rates herself as $5 / 10$ on 'degree of safety while driving,' where 10 is 'extremely safe.'

-She would 'probably agree' with the statement that her physician should be concerned about how well she drives and that she would follow her doctor's advice about driving.

\begin{tabular}{lc} 
Test Domain & Quintile $(5=$ worst $)$ \\
Visual Closure & 4 \\
Reaction Time & 3 \\
Visual Memory & 3 \\
Trailmaking & 3 \\
Directional sense & 5 \\
Attention & 5 \\
Self-report & Number reported \\
Crashes in last 2 years & 1 \\
Near misses in last 2 years & 12 \\
Mini-Mental State Examination & 25 \\
\hline
\end{tabular}

interpretable than the raw test results. The focus groups were audiotaped, and the tapes were transcribed. We searched the transcripts for recurrent themes related to barriers to assessing driving ability and opportunities for improving the assessment process according to standard methods. ${ }^{8}{ }^{89} \mathrm{We}$ also analyzed physicians' responses to presentation of neuropsychological data from patients, and we report here the themes that emerged in these meetings.

\section{Results}

A total of 20 physicians participated in one of 2 focus groups. A number of key themes raised by family physicians related to barriers to assessing driving in primary care. Here we provide a summary of the themes and examples from the transcripts. First, a concern that the physician will be liable for the results of driving-related screening, both testing that misses an impaired driver and testing that inappropriately classifies an older driver as impaired. For example, one physician described experience with the liability issue with regard to a specific patient: "He was a diabetic who drove off the beltway and hit a guardrail, and there was a guy whose car had broken down and the guy was sitting on the guardrail figuring he would be safe there and this guy hit him and was killed... The only thing that made me feel insulated from the whole thing is that he was not under my direct care at the time."

Second, physicians are not sure of their role in testing driving ability and feel obligated to inform the Motor Vehicles Administration of abnormal test results: "If there is any question you need the driving test and that should be conducted by the motor vehicle people, not us."

Third, patients will be defensive or angry at the physician. "Basically if I find someone who is not doing as well, I ask about their driving, how they are driving, but what I find is a lot of defensiveness, with people who are still driving trying to hold on to that." "It is very hard for physicians to say hey I need to test your cognitive abilities-there is a threat to that and it is almost easier when the family says, 'Hey, test Grandpa'.”

Finally, the available tests do not have adequate predictive ability.

Several key themes were raised by family physicians related to opportunities for assessing driving 
in primary care. First, driving ability should be a concern when primary care physicians evaluate patients. "We offer all sorts of guidance to the family that do or don't fall within the confines of what the doctor does, because you become someone this person considered to have some expertise at dealing with the family, not just the medical aspects."

Second, when the question of driving ability is raised by the patient, the family, or someone else, it would be valuable to have a set of recommended procedures to follow that could be performed in the office. "Basically, we haven't had a tool; if we have a tool now, then you've got to look not at just the sensitivity and the specificity of the tool. Because if you've got a tool that reliably predicts that people are impaired in their driving, then the implication would be that they should not drive. I think in terms of telling people to stop driving, I think that if you've got. . . more than $5 \%$ [false positives], you are going to be in the range of test that's not going to be of much practical value from the standpoint of trying to sell this to the public."

Third, testing can help identify someone who might be at risk for a crash in the near future. Fourth, tests that are related to driving performance would be useful, because most clinical testing is of limited value, and raising the question of driving in the context of the medical and functional evaluation prompts older patients to consider their own driving habits and safety behind the wheel; for example, asking direct questions about near misses and crashes. "I guess what may not be happening, which we may not be doing as part of our care evaluation, is asking about driving. I usually know if they are driving or not, but I very rarely ask about accidents."

There was general consensus on most points. However, a particularly contentious area was whether physicians should screen older persons for driving ability in the absence of specific concerns raised by the family or because of a crash. Physicians uniformly agreed that a protocol to guide assessment that contained tests that correlated with driving performance and that could be conducted in the office setting would be a valuable tool.

\section{Discussion}

The physicians who participated in the focus groups on driving and the elderly drew attention to several barriers to the use of the primary care set- ting as a venue for the assessment of older drivers. At the same time, there was a clear recognition of the deficiencies of the standard physical examination in detecting adults likely to be at risk for crashes and the need for the use of tests that relate more directly to driving performance. Recommendations for office-based assessment of at risk motor vehicle injury for older drivers include static visual screening, auditory screening, MMSE, depression assessment, functional assessment, and review of alcohol use and medications. ${ }^{10-12}$ However, the neuropsychological tests that tap perceptual and attentional processes that may be more directly related to driving skill have been lacking in such recommendations. The development of a protocol for office-based assessment for driving was a major goal of the overall project, but it is unlikely that such a protocol would be implemented in the primary care setting unless primary care physicians thought the new tests would be useful in advising patients and their families.

Before further discussing our findings, the limitations of our study deserve comment. First, our results were obtained from a limited number of primary care sites whose physicians and patients might not be representative of most primary care practices. However, these primary care practices were not academically affiliated and were probably similar to other primary care practices in the region. Second, physicians' remarks reflected their perceptions of their practice and might not reflect the actual practice of these physicians. Third, our sample size is small because we did not have the resources to carry out focus groups at more sites.

Given the balance between maintaining independence and concern for public safety, ${ }^{13}$ at what point should physicians consider that the risk of driving is too great? What possibilities and problems exist in performing a driving assessment in primary care?

\section{Barriers to Addressing Driving in Primary Care}

A primary concern voiced by the participating physicians related to the extent to which they would be liable for decisions made based on their testing, both in terms of assessing a driver as fit to drive and in assessing a fit driver as not performing well enough to drive. The physician has 2 duties that are sometimes at loggerheads with each other: a private duty to maintain patient confidentiality and a public duty to warn. ${ }^{13}$ There are legal precedents for 
liability in cases in which there was foreseeable risk to the public, and physicians have been liable for failure to warn. In marginal cases, it is important to document the extent of disability. ${ }^{13}$

Physicians were also uncertain as to whether they were obligated to inform the Motor Vehicle Administration about older adults who failed the proposed driving performance tests. In some states, Alzheimer disease is a reportable condition requiring testing. ${ }^{13}$ State administrations of motor vehicles maintain medical boards that are sources of information regarding the need to report drivers.

Suggesting that the patient's driving ability is in question and requires testing raises a great deal of anxiety on the part of patients and within the physician-patient relationship. The ability to drive represents a type of freedom, and the loss of this privilege represents a restriction of independence. ${ }^{14}$ There was also a general concern that patients do not react favorably to testing of cognitive status in the primary care setting.

Physicians were concerned about the performance of the neuropsychological tests themselves; that is, that tests will be able to predict the risk of driving. Several aspects of neuropsychological function such as visual and auditory attention, have been found to correlate with driving performance, but it is unclear whether the results of neuropsychological tests at baseline predict driving performance. ${ }^{5,14-16}$ The copy polygon task of the MMSE has been found by some studies ${ }^{17,18}$ to be related to adverse events while driving but not by other studies. $^{3}$

\section{Opportunities for Addressing Driving in Primary Care}

Physicians recognized the importance of driving within the context of geriatric functional assessment. Every physician had the experience of having driving ability as the main reason for the patient visit but felt uneasy with the standard clinical examination as the only assessment tool. Physicians felt it would be valuable to have a set of recommended procedures to follow that could be performed in the office. It is critical that if the physician's office is a venue for assessing older drivers, then tests that are feasible for primary care and that are predictive of driving skill must be developed within the primary care setting.

\section{Conclusion}

How physicians should assess and manage driving problems of older adults remains an area for continued dialogue and research. Our work extends beyond the simple evaluation of the performance characteristics of tests in predicting which older drivers are at increased risk of accidents or near misses. ${ }^{5}$ From the perspective of society, however, we need to understand the acceptability and suitability of possible testing of older drivers in primary care settings to inform public policy. The current study complements the prior work in which we have sought the perspective of patients. The patient questionnaire data in the prior study showed an overall willingness on the part of patients to accept the results of neuropsychological tests as a basis for making decisions about changes in driving habits. ${ }^{7}$ The current study adds insight about driving issues from the perspective of providers. Physicians felt it would be valuable to develop a brief, effective, and feasible assessment strategy for evaluating older drivers in primary care. Future studies are needed to develop a clinically valid but practically feasible driving assessment tool.

The investigators thank the participating physicians and patients at the Family Health Center of the Franklin Square Hospital Center, Baltimore, Maryland, and the East Point Medical Center, Baltimore, Maryland.

\section{References}

1. Physicians' guide to driver examination, 5 th ed. Can Med Assoc J 1991;15 Suppl:1-64.

2. Folstein MF, Folstein SE, McHugh PR. "Mini-mental state." A practical method for grading the cognitive state of patients for the clinician. J Psychiatr Res 1975;12:189-198.

3. Gallo JJ, Rebok GW, Lesikar S. The driving habits of adults aged 60 years and older. J Am Geriatr Soc 1999;47:335-41.

4. Rebok GW, Keyl PM, Bylsma FW, Blaustein MJ, Tune L. The effects of Alzheimer's disease on driving-related abilities. Alzheimer Dis Assoc Disord 1994;8:228-40.

5. Lesikar SE, Gallo JJ, Rebok GW, Keyl PM. Prospective study of brief neuropsychological measures to assess crash risk in older primary care patients. J Am Board Fam Pract 2002;15:11-19.

6. Keyl PM, Rebok GW, Gallo JJ. Screening older drivers in general medical settings: toward the development of a valid and feasible assessment procedure. Final Report prepared for the American Association of Retired Persons. Andrus Foundation, December 1997. 
7. Deleted in proof.

8. Gubrium JF, Sankar A. Qualitative methods in aging research. Thousand Oaks (CA): Sage Publications; 1994.

9. Miles MB, Huberman AM. Qualitative data analysis: an expanded sourcebook, 2nd ed. Thousand Oaks (CA): Sage Publications; 1994.

10. Underwood $M$. The older driver: clinical assessment and injury prevention. Arch Intern Med 1992;152: 735-40.

11. Carr D, Rebok GW. The older driver. In: Gallo JJ, Fulmer T, Paveza GJ, Reichel W, editors. Handbook of geriatric assessment, 3rd ed. Gaithersburg (MD): Aspen Publishers; 2000. p. 149-157.

12. Carr D. Driving and the older adult. In: Gallo JJ, Busby-Whitehead J, Rabins PV, Silliman R, Murphy J, editors. Reichel's care of the elderly: clinical aspects of aging. 5th ed. Baltimore: Lippincott Williams \& Wilkins; 1999. p. 726-38.

13. Retchin SM, Anapolle J. An overview of the older driver. Clin Geriatr Med 1993;9:279-96.

14. Reuben D, Silliman R, Traines M. The aging driver: medicine, policy, and ethics. J Am Geriatr Soc 1988; 36:1135-42.

15. Marottoli RA, Richardson ED, Stowe MH, et al. Development of a test battery to identify older drivers at risk for self-reported adverse driving events. J Am Geriatr Soc 1998;46:562-8.

16. Sims RV, Owsley C, Allman RM, Ball K, Smoot
TM. A preliminary assessment of the medical and functional factors associated with vehicle crashes by older adults. J Am Geriatr Soc 1998;46:556-61.

17. Marottoli RA, Cooney LM, Wagner DR, Doucette J, Tinetti ME. Predictors of automobile crashes and moving violations among elderly drivers. Ann Intern Med 1994;121:842-6.

18. Hunt L, Morris JC, Edwards D, Wilson BS. Driving performance in persons with mild senile dementia of the Alzheimer type. J Am Geriatr Soc 1993;41: 747-53.

19. Hammill DD, Colarusso RP. The motor-free visual perception test. Novato (CA): Academic Therapy Publications; 1972.

20. Huhtaniemi P, Haier RJ, Fedio P, Buchsbaum MS. Neuropsychological characteristics of college males who show attention dysfunction. Percept Mot Skills 1983;57:399-406.

21. Wechsler D. Manual for the Wechsler Memory Scale - revised (WMS-R). New York: The Psychological Corporation; 1987.

22. Reitan RM, Wolfson D. The Halstead-Reitan neuropsychological test battery. Tucson: Neuropsychology Press; 1958.

23. Money JA. A standardized road map of directional sense, manual. San Rafael (CA): Academic Therapy Publications; 1976.

24. Schretlen D. The brief test of attention. Baltimore: Psychological Assessment Resources; 1989. 